\title{
LA EVALUACIÓN DE LOS APRENDIZAJES EN LA ENSEÑANZA CLÍNICA DE LA ENFERMERÍA.
}

\author{
EVALUATION OF LEARNING IN CLINICAL OF NURSING EDUCATION.
}

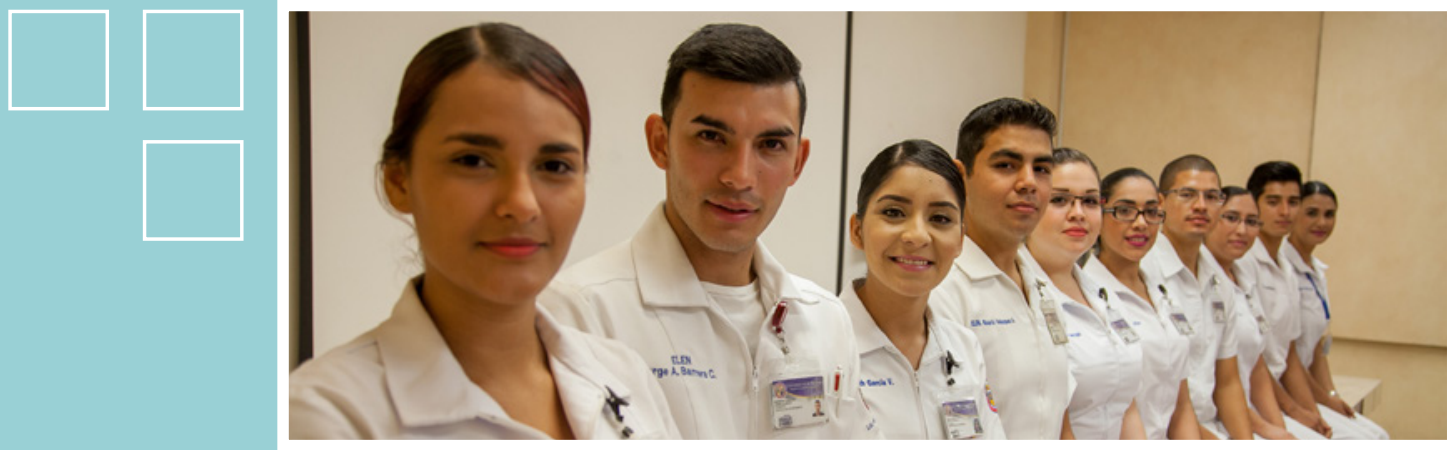

\section{ABSTRACT}

This article discusses the ways of evaluation by the teaching staff in the field of clinical practice in nursing, and the teacher is the one who develops strategies of teaching - as needed basis, to guide students to make their own learning for development of a professional future. It aims to see in a general way the process of learning assessment in clinical practice that made teachers summarized in three categories: academic performance of students, evaluation of products (results of teaching), and search standardizable attributes or traits, these methods are adapted according to the clinical area where the student develops.

It can be an excellent professional without being therefore an excellent teacher and therefore no longer be an excellent evaluator that considers three main causes of errors in the evaluation process: Insufficient information halo effect, or tendency to establish categories and stereotypes error generosity, giving them a preamble to a hazard assessment invalid and unreliable bringing consequences of psychological, physical and social environment for nursing students.

Keywords: evaluation, clinical practice, teaching - student nurse, nursing teacher.

\section{RESUMEN:}

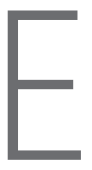
I presente artículo aborda las formas de evaluación por parte del personal docente en el ámbito de práctica clínica en Enfermería, ya que el docente es quien desarrolla estrategias de enseñanza - aprendizaje, como bases necesarias, que guiarán al estudiante a realizar su propio aprendizaje para el desarrollo de un futuro profesional. Se pretende ver de una manera general el proceso de evaluación de los aprendizajes en práctica clínica que realizan los docentes que se resume en tres categorías: El rendimiento académico de los estudiantes, la evaluación de productos (resultado de la enseñanza), y la búsqueda de atributos o rasgos estandarizables que son adaptados según el área clínica donde se desenvuelve el estudiante. Un profesional puede ser excelente en su trabajo sin ser un excelente profesor, tampoco será un excelente evaluador ya que se consideran cuatro principales causas de error en el proceso de evaluación: información insuficiente, efecto de halo, tendencia a establecer categorías o estereotipos y error por generosidad, dando éstos un preámbulo para un riesgo de una evaluación no válida y no confiable produciendo consecuencias del ámbito psicológico, físico y social para los estudiantes de enfermería.

$\left(^{*}\right)$ Doctora en Educación. MTC Titular B Depto. de Enfermería UNISON

(**) Doctora en Educación. MTC Titular B Depto. Enf. UNISON
Palabras clave: Evaluación, práctica clínica, enseñanza - aprendizaje, estudiante de enfermería, docente de enfermería. 


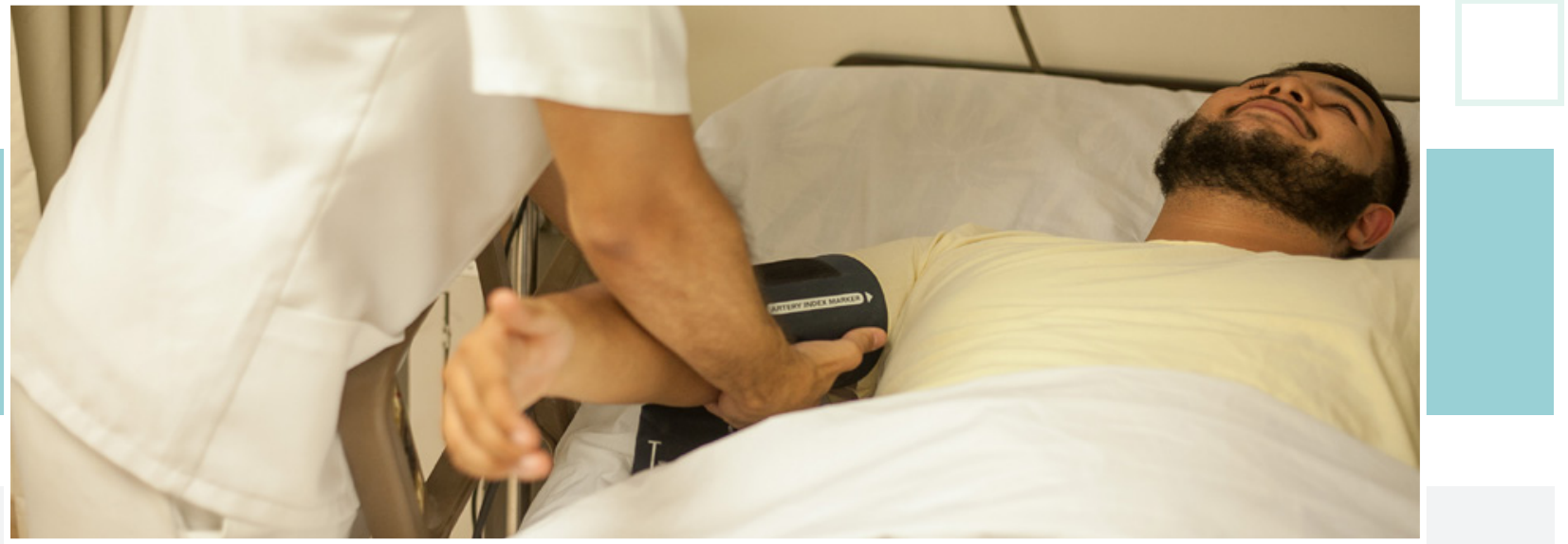

\section{INTRODUCCIÓN}

La evaluación, es un aspecto crítico en todo análisis de la enseñanza aprendizaje, debe ser planteada de manera congruente y una de las finalidades de la enseñanza en enfermería es contribuir a lograr competencias para la vida en el mundo actual, competencias para abordar situaciones nuevas y responder a los retos de la práctica profesional, adquiridas por su propia vivencia (1).

Una dificultad para evaluar los aprendizajes reside en que los criterios de evaluación varían de acuerdo al área en la que realizan la práctica clínica los estudiantes. Los porcentajes que se asignan a los aprendizajes (cognitivos, motrices y socio afectivos), son determinados por los profesores quienes frecuentemente difieren en relación a criterios e instrumentos de evaluación, incluso los que supervisan la misma asignatura en grupos diferentes.

\section{PROPÓSITO}

En las siguientes líneas se pretende plantear una visión integral del proceso de evaluación de los aprendizajes en la práctica clínica que realizan los profesores en una Escuela de Enfermería y también entender por qué los estudiantes muestran angustia cuando al término de cada semestre serán evaluados sus logros académicos en la práctica que realizan en las diferentes instituciones a las que asisten.
El presente escrito tiene como actores principales a los docentes y estudiantes quienes participan en el proceso de enseñanza aprendizaje en un ámbito en el que los alumnos tendrán que demostrar competencias que incluyen los saberes cognitivos, motrices y socio afectivos.

\section{LA EVALUACION}

Son muchas las discusiones que se realizan entre diferentes teorías acerca de las relaciones entre objetivos y evaluación. Si entendemos que los objetivos son los resultados esperados al finalizar el proceso de enseñanza y aprendizaje, es lógico pensar que estos últimos se conviertan en el qué de la evaluación. El docente tiene que evaluar aquello que propuso como resultado del aprendizaje tras haber vigilado lo que hace y cómo lo hace mediante supervisión y asesoría(2). Para valorar el aprendizaje de los alumnos, los docentes pueden recopilar información acerca de los conocimientos, las actitudes y las destrezas de los alumnos para poder llevar a cabo una evaluación integral.

Desde el punto de vista histórico es bien sabido que existe un gran conflicto en relación a cómo, cuándo y qué evaluar durante el proceso de enseñanza aprendizaje. En la práctica de la enseñanza de la enfermería se observa que en los resultados de la evaluación, raramente existe alguna correlación entre lo evaluado y lo que se quería medir y conseguir en relación al producto profesional; es decir no se conoce la validez de dichas evaluaciones ya que se da un sesgo al dar cifras que se pueden multiplicar, dividir y transformar en valores estadísticos. 


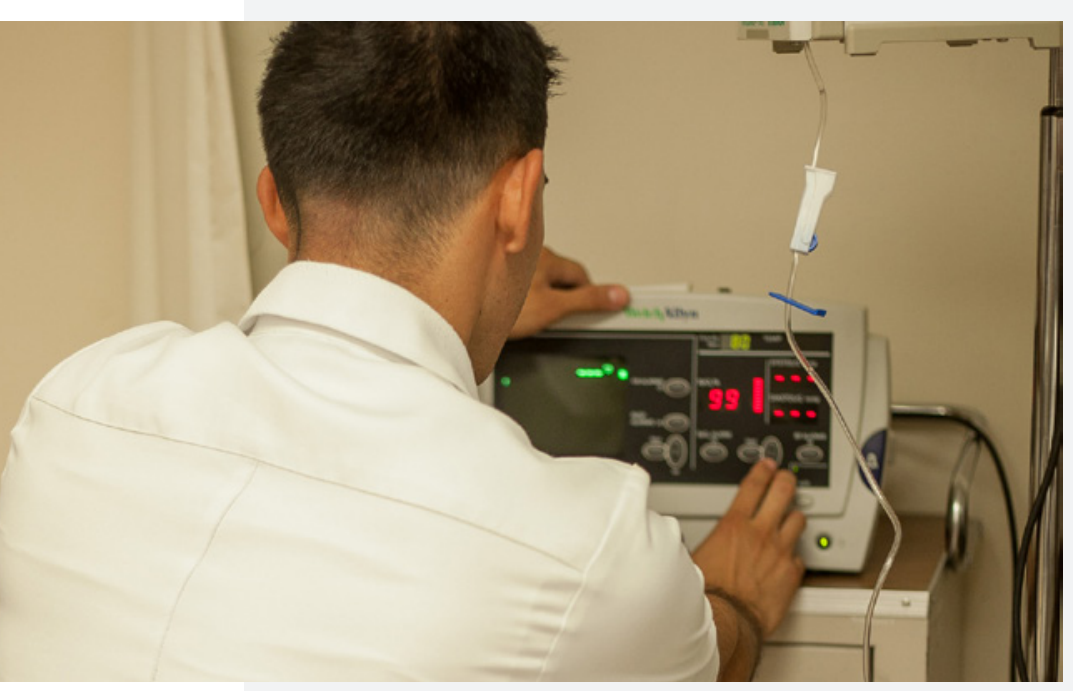

dar cifras que se pueden multiplicar, dividir y transformar en valores estadísticos.

Es necesario mantener claro que hay razones y responsabilidades docentes y de la institución educativa para que el proceso de evaluación se cumpla. El no hacerlo o hacerlo parcialmente hace manifiesto que los docentes no reconozcan que la sociedad espera más de los profesionales de la enfermería y que es fundamental que el docente recuerde en forma constante, que el proceso de evaluación de los aprendizajes determina en forma acentuada los métodos o estrategias de aprendizaje que el estudiante utiliza durante su formación académica.

\section{LA EVALUACIÓN COMO ELEMENTO DEL MODELO DIDÁCTICO}

La evaluación es un aspecto que tiene que mantenerse en estricta dependencia respecto de los elementos didácticos, es la comprobación en el planteamiento de la validez de las estrategias didácticas, configuradas por las opciones que se han tomado en las numerosas dimensiones de los elementos didácticos, en orden de consecución de los objetivos propuestos en un programa de enseñanza. Es decir, es el momento de buscar los datos que faciliten la toma de decisiones del profesor y que permitan visualizar si la estrategia metodológica desarrollada es adecuada o no, o en que medida lo es, para guiar un proceso de aprendizaje que aterrice en la obtención de unos resultados de aprendizaje previamente propuestos. ${ }^{(3)}$
En la enseñanza de la enfermería en la evaluación de los aprendizajes se ha utilizado dentro del proceso tradicional solamente con funciones de valoración cuantitativa y aquí cabría hacer la pregunta qué función tiene este concepto de evaluación externa al propio proceso de enseñanza aprendizaje y sin relaciones con él.

Es bien sabido que la evaluación es una parte sustancial de todo programa educativo y se concibe como un proceso continuo que da cuenta de las acciones pedagógicas planeadas por los docentes y de los resultados obtenidos por los estudiantes, esto nos permite afirmar que el proceso no es unidireccional, el docente es quien realizará los ajustes pertinentes mediante razonamientos, pautas de conducta, demostraciones, y otros medios, de tal manera que los resultados sean los esperados o lo más cercano a lo planeado. ${ }^{(4)}$

Es importante que los docentes adopten ciertos criterios, instrumentos y procedimientos para valorar la congruencia de los propósitos, los contenidos y las actividades que los programas de materias prácticas de enfermería sugieren con el fin de sustentar la toma de decisiones para realizar una evaluación objetiva, pertinente, justa $y$, no centrar la evaluación en cogniciones aisladas sin considerar su conexión con el marco de conocimientos general y personal del alumno, esta tendencia a evaluar conocimientos como unidades ordenadas que se pueden aislar en forma superficial, no favorece la construcción del conocimiento ni la vinculación teoría práctica que considere la articulación de las cadenas entre conocimiento, aplicación práctica y cambio social, además el aprendizaje que puede ser modificado, siendo una responsabilidad de los docentes ayudar a los estudiantes a descubrir su estilo y aprender a adaptarlo a las experiencias de cada situación. (5)

\section{LOS MÉTODOS}

Para evaluar los aprendizajes de los estudiantes los métodos más utilizados por los profesores son los que centran su atención en las siguientes direcciones:

El rendimiento académico de los estudiantes, la evaluación de productos (resultado de la enseñanza), y la búsqueda de atributos o rasgos estandarizables. 
Los procedimientos implementados por los docentes para evaluar los aprendizajes en las materias prácticas, varían en función de los criterios muy personales de los profesores y son adaptados de acuerdo al área en la que realizan la práctica clínica los estudiantes; Las ventajas de la educación basada en las competencias del estudiante de enfermería potencia la responsabilidad del alumno haciéndolo partícipe de manera activa en su proceso de aprendizaje ya que las competencias del alumno determinan aquello que deben aprender los estudiantes y aquello que debe ser evaluado(6). Los porcentajes que se asignan a los aprendizajes (cognitivos, motrices y socio afectivos), son determinados por los profesores quienes difieren en relación a esos criterios e instrumentos de evaluación incluso los que imparten la misma asignatura en grupos diferentes, como se podrá ver los maestros tienen patrones de medida que restan exactitud a las calificaciones y son controladas por las apreciaciones muy particulares.

\section{LAS APRECIACIONES PERSONALES DEL PROFESOR}

Cuando el profesor califica a un alumno, es frecuente que recurra a apreciaciones personales. Aunque muchas veces estos juicios estimativos presentan mayor valor predictivo que las calificaciones obtenidas por instrumentos objetivos, también pueden encontrarse viciadas por un cúmulo de factores psicológicos que actúan como causas de error.

Las estimaciones de los profesores tienen las siguientes características ${ }^{(7)}$ :

- Son generalmente demasiado altas.

- Tienden a sobreestimar los logros de los alumnos débiles y a subestimar los de los más capaces.

- $\quad$ Los patrones de medida utilizados varían considerablemente de un profesor a otro.

La facultad de emitir juicios es el símbolo de la autoridad encarnada por el profesor e implica tantos riesgos que conviene analizar las posibles causas de error operantes en la elaboración de juicios estimativos sobre la actuación del alumno. Se puede ser un excelente profesional sin ser por eso un excelente profesor y por consiguiente tampoco será un excelente evaluador porque el objetivo central del proceso de evaluación es facilitar la tarea del estudiante, es decir, que pueda precisamente alcanzar sus propias metas y objetivos(8).

El docente debe de ser capaz de utilizar adecuadamente las nuevas metodologías, de manera que sea competente para desarrollar sus capacidades de procesamiento, diagnóstico, decisiones, evaluación de procesos, reformulación de proyectos y génesis de pensamiento práctico en los estudiantes(9). A continuación veamos algunas de las causas de errores en la evaluación:

Información insuficiente: el profesor no cuenta a menudo con suficientes evidencias acerca del desempeño del estudiante como para poder calificarlo de manera justa. En la práctica cotidiana durante la supervisión de los alumnos en los campos clínicos el profesor evalúa el rendimiento de los alumnos en períodos muy cortos; es decir el número de veces que logra observarlo es mínimo, por lo que sus juicios concluyen siendo frecuentemente improvisados, y por lo tanto la información recabada acerca del rendimiento de los estudiantes resulta a todas luces incompleta lo que favorece que se emitan juicios sobre bases inseguras, influidos más por circunstancias azarosas que por una cuidadosa ponderación de los aciertos y errores de los estudiantes.

Efecto de Halo: "Es la tendencia a formar una impresión o juicio sobre una característica particular de un individuo

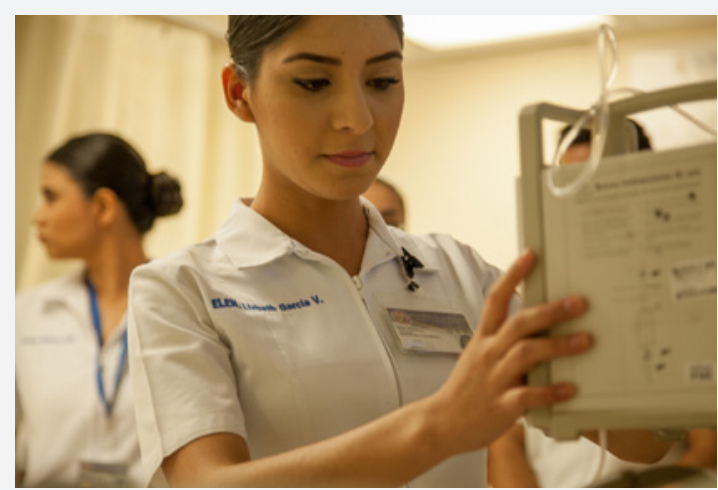


basándose en la impresión general que se tiene de él. Si la impresión general es favorable será mejor calificado. Este aspecto favorece o repercute de manera negativa en los estudiantes ya que la evaluación actúa como un prejuicio que impide la valoración objetiva del rendimiento y por consiguiente se distorsiona el resultado.

\section{Tendencia a establecer Categorías} o Estereotipos: la categorización ayuda a los profesores que tienen que evaluar a grupos grandes de estudiantes, como es el caso de los grupos de estudiantes de enfermería de la Universidad pública, a quienes se les percibe como pertenecientes a un tipo determinado y se les atribuyen rasgos característicos del grupo al que pertenecen. La base de las categorizaciones está influida por los estereotipos o también por la "etiqueta" que se coloca al estudiante y que semestre tras semestre tiene que cargar con ella. Son como las Ilama Hofstätter(10), "soluciones patentadas" que liberan de la penosa inseguridad que ataca al profesor que debe calificar a grupos saturados en un tiempo limitado y por consiguiente al estudiante que le tocó la suerte de estar en alguna categoría preestablecida por el profesor será evaluado no en base a los aprendizajes sino más bien en base a los juicios y percepciones del docente.

Error por generosidad: Es común que los profesores que supervisan las prácticas clínicas se sientan inseguros al asignar una calificación para el alumno. Esta inseguridad se deriva de la indeterminación del significado atribuido a cada uno de los elementos que se evalúan y los puntajes asignados a cada rubro; la indefinición de los objetivos cuyo logro se debe evaluar, o en la falta de exactitud de las medidas obtenidas por medio de técnicas de evaluación en las que no se confía totalmente, los estudiantes frecuentemente se quejan de que en un semestre los profesores los supervisan sólo en dos o tres ocasiones y con esos elementos serán evaluados. Con todas estas notas en mente, y sabiéndose juez y parte al mismo tiempo, no es raro que tienda a calificar generosamente a los estudiantes. Esta situación bien puede favorecer al estudiante pero también puede haber una calificación desfavorable que puede afectar seriamente al alumno y entonces sucede lo contrario, en este caso se genera un error por mezquindad. Atribuido a lo anterior, en ocasiones la evaluación al término del ciclo escolar es una realidad que genera desconcierto, temor y angustia en los estudiantes antes de ser evaluados ${ }^{(3)}$.

\section{DEBATE}

La evaluación de los aprendizajes de los estudiantes de enfermería en las materias prácticas se ubica dentro del proceso de enseñanza aprendizaje tradicional ya que la función es meramente cuantitativa y solamente controla el conocimiento al final del proceso.

Se reconoce que en los procesos de enseñanza aprendizaje intervienen profesoresyestudiantes, en el programa curricular planteado desde un modelo pedagógico específico, el único sujeto evaluable del sistema universitario es el alumno, siendo que la evaluación debe ser un medio para comprender no sólo los resultados logrados por los alumnos, sino también para alcanzar la validación y análisis de estrategias metodológicas, entonces el problema de los resultados del proceso de enseñanza aprendizaje hay que enfocarlo de una manera holística y no sólo en términos de objetivos explícitamente propuestos de ahí que surge si es o no correcta la manera de enseñanza del personal docente(11).

La acción pedagógica por su carácter totalizador, trae consecuencias a veces nefastas por los efectos que produce en los estudiantes. Se identifican las siguientes características del que evalúa: hay autoritarismo y subjetividad que lleva a tomar decisiones basadas en la fijación de observaciones imprecisas. El peligro de la evaluación no válida y no confiable conlleva a la acentuación de consecuencias psicológicas, físicas y sociales de los estudiantes de enfermería.

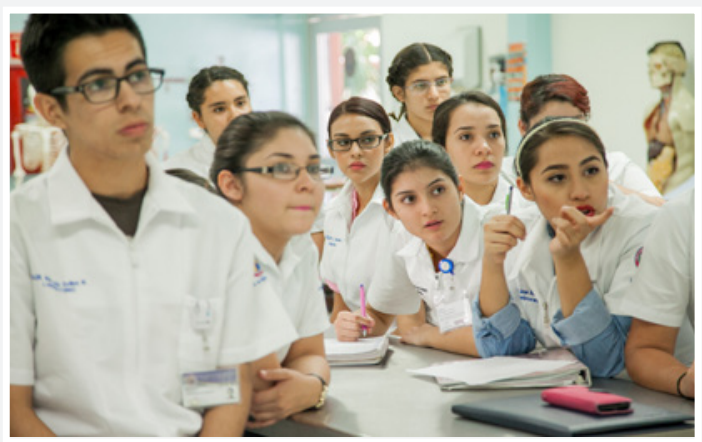




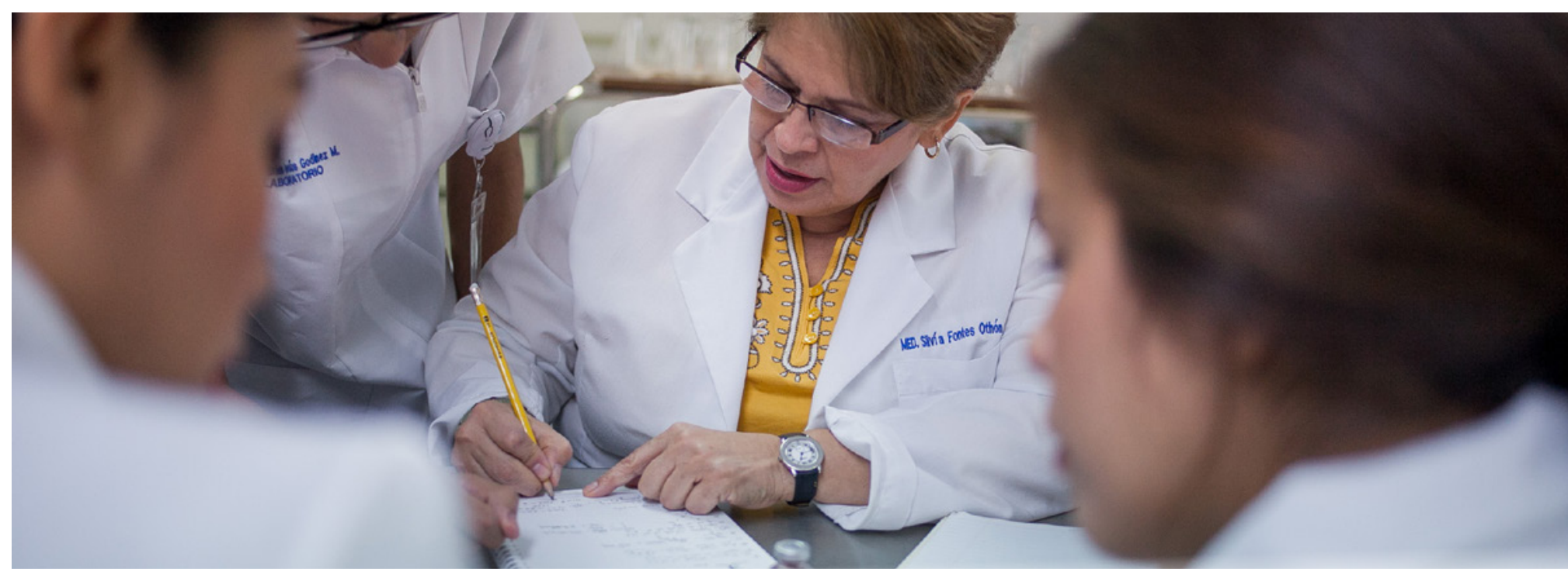

\section{REFERENCIAS BIBLIOGRÁFICAS}

1. Bettancourt L, Muñoz LA, Merighi MAB, dos Santos MF. El docente de enfermería en los campos de práctica clínica: un enfoque fenomenológico. 2011.

2. Lifshitz A. IV. La enseñanza de la competencia clínica. Gaceta médica de México. 2004;140(3):312-3.

3. Casanova MA. Manual de evaluación educativa: La muralla; 2002.

4. CastaÑeda Figueiras S. Evaluacion Del Aprendizaje en El Nivel Universitario. Elaboracion: UNAM; 2006.

5. Canalejas Pérez M, Martínez Martín M, Pineda Ginés $M$, Vera Cortés $M L$, Soto González $M$, Martín Marino Á, et al. Estilos de aprendizaje en los estudiantes de enfermería. Educación médica. 2005;8(2):33-40.

6. Falcó Pegueroles A. La nueva formación de profesionales: sobre la competencia profesional y la competencia del estudiante de enfermería. Educación médica. 2004;7(1):42-5.
7. McClelland KA. Self-conception and life satisfaction: Integrating aged subculture and activity theory. Journal of Gerontology. 1982;37(6):723-32.

8. Ayala-Valenzuela $R$, Torres-Andrade $M$. Didáctica de la enseñanza: prácticas ejemplares en el sector salud. Educación Médica Superior. 2007;21(2):0-.

9. Quiles ÁS, Riera JRM. Nuevo enfoque en el proceso de enseñanza-aprendizaje para la interrelación de conocimientos y formación de clínica/comunitaria. Investigación y educación en enfermería. 2008; 26(2):150-9.

10. Hofstätter PR. Introducción a la psicología social: Luis Miracle; 1966.

11. Crespo Knopfler S, González Velázquez MS, Agama Sarabia A. Percepción de los alumnos de enfermería sobre los factores que inciden en su aprendizaje clínico. Vertientes Revista Especializada en Ciencias de la Salud.14 ${ }^{(2)}$. 